\title{
Transformation of a wave energy spectrum from encounter to absolute domain when
} observing from an advancing ship

\author{
Nielsen, Ulrik Dam
}

Published in:

Applied Ocean Research

Link to article, DOI:

10.1016/j.apor.2017.10.011

Publication date:

2017

Document Version

Peer reviewed version

Link back to DTU Orbit

Citation (APA):

Nielsen, U. D. (2017). Transformation of a wave energy spectrum from encounter to absolute domain when observing from an advancing ship. Applied Ocean Research, 69, 160-172.

https://doi.org/10.1016/j.apor.2017.10.011

\section{General rights}

Copyright and moral rights for the publications made accessible in the public portal are retained by the authors and/or other copyright owners and it is a condition of accessing publications that users recognise and abide by the legal requirements associated with these rights.

- Users may download and print one copy of any publication from the public portal for the purpose of private study or research.

- You may not further distribute the material or use it for any profit-making activity or commercial gain

- You may freely distribute the URL identifying the publication in the public portal

If you believe that this document breaches copyright please contact us providing details, and we will remove access to the work immediately and investigate your claim. 


\title{
Transformation of a wave energy spectrum from encounter to absolute domain when observing from an advancing ship
}

\author{
Ulrik D. Nielsen ${ }^{\mathrm{a}, \mathrm{b}}$ \\ ${ }^{a}$ DTU Mechanical Engineering, Technical University of Denmark, DK-2800 Kgs. Lyngby, Denmark \\ ${ }^{b}$ Centre for Autonomous Marine Operations, AMOS-NTNU, NO-7491 Trondheim, Norway
}

\begin{abstract}
The article presents a practical approach to transform a wave energy spectrum from encounter domain to absolute domain. This problem has its specific relevance, when shipboard sea state estimation is conducted by the buoy analogy; notably for some particular implementation solving for the sea state directly in the encounter domain. The encounter domain is that observed from a ship when it advances in a seaway, whereas the absolute domain is that corresponding to making observations from a fixed point in the inertial frame. Spectrum transformation can be uniquely carried out if the ship sails "against" the waves (beam to head sea) but in following sea conditions there exists no unique solution to the problem. Instead, a reasonable approach valid for practical engineering must be applied, and the article outlines one viable solution that can be used to transform a wave spectrum from encounter to absolute domain. Specifically, two pseudo algorithms are presented, and good performance is achieved with both algorithms when they are tested at different operational scenarios.
\end{abstract}

Keywords:

Wave spectrum, advancing ship, spectrum transformation, encounter domain, absolute domain

\section{Introduction}

Today, marine vessels are heavily equipped with measuring sensors, and vast amount of information can be extracted by analysing the associated time history recordings.

Email address: udn@mek.dtu.dk (Ulrik D. Nielsen)

Preprint submitted to Applied Ocean Research

August 8, 2017 
Typical 'observing sensors' are motion response units (MRUs), hull girder measuring devices (like strain gauges), and sea state recorders. Making a note on the latter, one means of a sea state recorder on a ship is given in terms of the wave buoy analogy [? ]. With this analogy, sensor measurements of ship motions, or other global ship responses such as hull girder stresses, can be used to infer about the on-site sea state, in a similar way as is done with traditional floating wave buoys. Often, a vessel's measuring system and its sensors are part of an on-board decision support tool used to provide advice on speed and relative wave heading to the vessel's crew, and/or the monitoring system may be a component of larger shore-based measuring campaign applied to aid in strategic and business-related longer-term decisions.

Analyses of the measured wave-induced motions and other types of (structural) responses of a ship in a seaway can be made with few complications if the objective is merely to calculate statistics of the past measurements; usually carrying out the analyses by spectral analysis. Similarly, any observation of the wave system can be easily processed and analysed if interest concerns only the encountered wave energy distribution, equivalently wave spectrum. Here, it is understood that the 'encounter domain' is that one observed from the advancing ship, and it is assumed that advance speed and heading relative to the wave system do not change while observing. However, if realtime operator guidance with respect to (optimum) vessel speed and wave heading is in study, there is a need to transform the encountered wave energy spectrum to the ("true") absolute domain; which is the domain any fixed observer without advance speed, relative to the inertial frame, is in. Thus, if not this transformation to absolute domain is made, it will not be possible to evaluate the effect of heading and/or speed changes of the vessel. The fundamental mathematics describing the problem - transforming from the encounter domain to the absolute domain, or vice versa - is basically governed by the Doppler Shift together with a requirement imposing energy conservation. If spectral analysis is carried out, the conservation of energy implies that corresponding sets of frequencies in the two domain map identical amounts of energy, as illustrated in Figure 1.

From a theoretical point-of-view, the transformation problem is elementary, and most standard textbooks on naval architecture [? ? ? ? ] consider the topic of a spectrum transformation for a ship advancing forward with constant speed and at fixed heading 

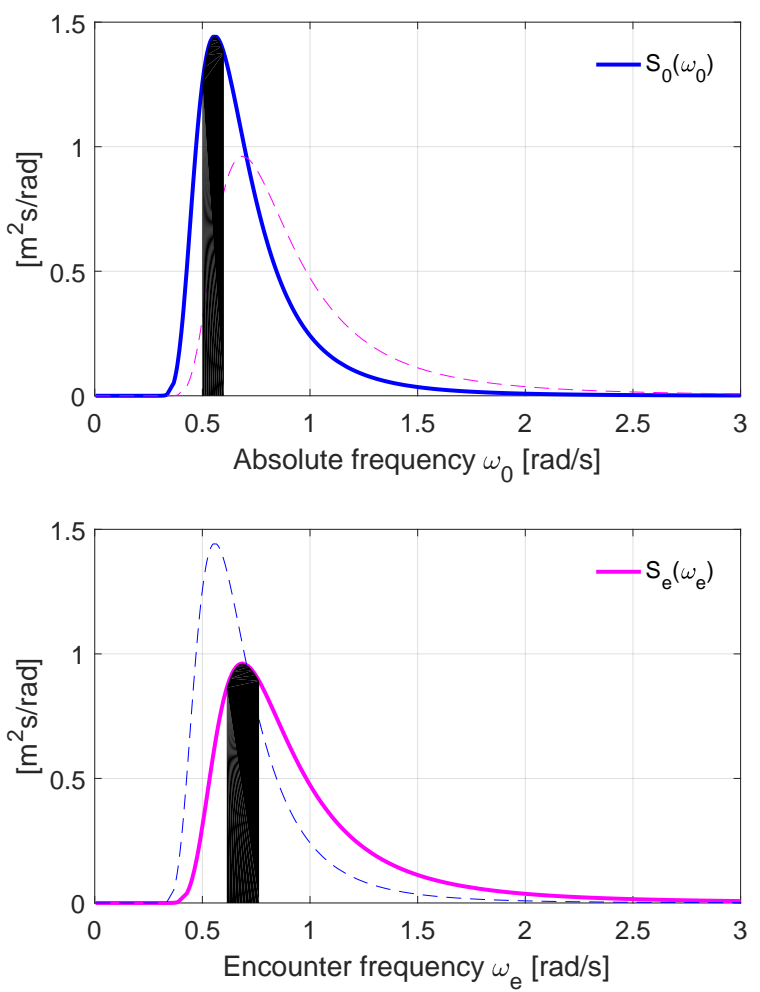

Figure 1: Energy is conserved for corresponding set of frequencies in the absolute domain and the encounter domain for an observer moving at 10 knots against the waves at a 30 deg. angle to the incoming waves. The distribution of wave energy follows a Bretschneider (Eq. 23) wave spectrum with significant wave height $H_{s}=3.0 \mathrm{~m}$, and zero-upcrossing period $T_{z}=8.0 \mathrm{~s}$.

relative to the waves that may be travelling in any direction. In practice, on the other hand, the problem is not easy to correctly address. Thorough and worthwhile readings on the topic are given by [? ] and [? ], where notably the latter points out the practical 'considerable complication' involved when transforming a (wave) spectrum. Nonetheless, both references have some detailed discussions and contain sort of "recipes" on how to transform a spectrum from the absolute domain to the encounter domain, but, disappointingly, they do not consider the reverse transformation - from encounter domain to absolute domain - and neither does other literature seem to practically address the problem.

This article provides two possible solutions, given in terms of pseudo algorithms, which assures that the - from an advancing ship - observed wave energy spectrum is 
properly transformed from encounter domain to absolute domain. In the broader context, this problem and the corresponding solution have their specific interest, or their origin and necessity, when shipboard sea state estimation (SSE) is conducted by the wave buoy analogy; notably for some particular implementations of the wave buoy analogy when it is applied and solved for directly in the encounter domain using spectral analysis to carry out the fundamental (and initial) response analysis. For instance, this is the case with over-the-bow-looking wave recorders that basically combine sensor readings from a relative motion sensor and an accelerometer to provide the encountered wave elevation record that, subsequently, can be used to derive the single-point-wave spectrum [? ? ]. The particular spectrum applies to the encounter domain and, as the final step and taking the relative heading between waves and vessel to be known, it is therefore necessary to transform to the absolute domain. More recently, the interest in a consistent transformation procedure has occurred due to research in a new implementation of the wave buoy analogy. The initial work [? ] has considered vessels without advance speed, as focus was on ships being dynamically positioned. In this case, 'encounter domain' is equivalent to absolute domain and, hence, transformation is unnecessary, but a generalisation of the implementation, [? ], for forward-moving ships requires indeed the estimated spectrum to be transformed from encounter to absolute domain. Moreover, newer but still quite conceptual studies, see [? ], will also necessitate a way to carry out consistently the transformation of the wave energy spectrum.

The suggested transformation-procedure applies specifically to problems formulated through spectral analysis and concerns transformation in the one "direction" only; from encounter domain to absolute domain. This choice is made because of the specific context mentioned above. Apart from that, transformation in the other direction can be uniquely carried out [? ? ] although, when the ship advances in following waves, generally the calculated encounter-wave spectrum can be heavily distorted, since three absolute-wave components map simultaneously into one encounter-wave component for some conditions. However, in the transformation from encounter to absolute domain there exists no unique solution on how to distribute the energy of an encounter-wave component to the three corresponding absolute-wave components, when the ship advances in following waves. These considerations should become more clear after reading 
the article. It is noteworthy that the presented solutions and the associated algorithms have focus on long-crested waves only, but this restriction can be relaxed. Moreover, deep-water conditions are assumed throughout.

\subsection{Composition of the article}

The paper is organised into 6 sections. Following the Introduction, Section 2 lays out the theoretical formulations related to spectrum transformation and the section brings forward the practical complications. In Section 3, two suggested solutions are presented in terms of (pseudo) algorithms to be applied when a wave energy spectrum needs to be transformed from the encounter domain to the absolute domain. Section 4 introduces a number of case studies upon which the performance of the transformation algorithm(s) can be tested, and the associated results and discussions are contained in Section 5 . Finally, Section 6 puts down the conclusions from the study.

\section{Ocean wave spectrum transformations}

The transformation of wave energy spectra from the one domain to the other relies fundamentally on the fact that energy, totally, must be conserved. Thus, the governing theoretical formulation reads

$$
S_{e}\left(\omega_{e}\right) d \omega_{e}=S_{0}\left(\omega_{0}\right) d \omega_{0}
$$

where $\omega_{e}$ and $\omega_{0}$ are corresponding pairs of encounter frequencies and absolute wave frequencies, respectively, while $S_{e}$ is the wave spectral ordinate in the encounter domain whereas $S_{0}$ is the spectral ordinate in the absolute domain. The energy equivalence, expressed by Eq. (1), was illustrated in Figure 1, where a Bretschneider wave spectrum (significant wave height $H_{s}=3.0 \mathrm{~m}$, zero-upcrossing period $T_{z}=8.0 \mathrm{~s}$ ) was transformed from the absolute domain to an encountered domain observed on a ship advancing with speed $U=10$ knots at heading $\beta=150 \mathrm{deg}$. relative to the wave system. Note, $\beta=180$ deg. corresponds to head sea, and $\beta=0 \mathrm{deg}$. is following sea.

The mathematical relation between encounter frequency $\omega_{e}$ and absolute (wave) frequency $\omega_{0}$ depends on the vessel's speed $U$ and heading $\beta$ relative to the waves. The 


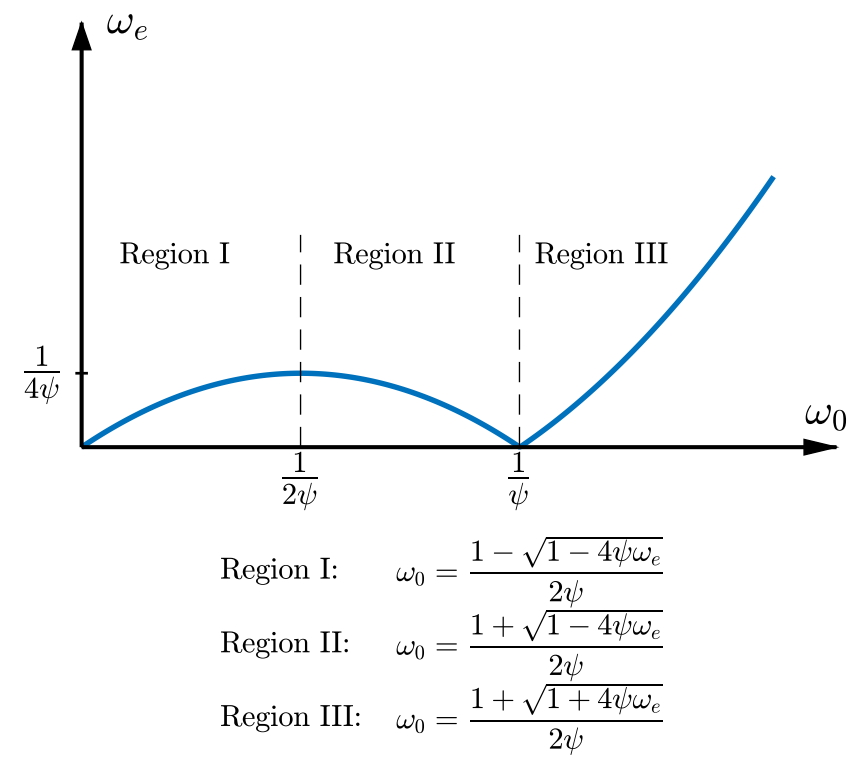

Figure 2: The relationship between encounter wave frequencies and absolute ("true") wave frequencies is governed by the Doppler Shift leading to a parabola. If $\omega_{0}$ is solved for $\omega_{e}$, three solutions need to be considered. $(\psi=U / g \cos \beta)$

relation is for deep-water conditions given by,

$$
\omega_{e}=\omega_{0}-\omega_{0}^{2} \psi, \psi=\frac{U}{g} \cos \beta
$$

which is the well-known Doppler Shift that applies whenever the observer moves relative to the travelling waves; not limited to only ocean surface waves but all kinds of travelling waves transporting energy in a continuum. For an advancing vessel in ocean waves it can be seen that for waves approaching from beam to bow (head sea), i.e. $\beta=[90-180]$, there is a 1-to-1 mapping of corresponding frequencies. Obviously, there is thus no difficulty in transforming a wave spectrum from the one domain to the other, independent on the direction of transformation.

In following waves, $\beta=[0-90[$, on the other hand, Eq. (2) represents (graphically) a parabola in the right-hand half of the $\left(\omega_{0}, \omega_{e}\right)$-plane. The parabola for following waves is illustrated in Figure 2, where it is noted that no such thing as a negative (encounter) frequency exists in the observed domain and, hence, the "negative leg" of the parabola has been turned upwards. This means that three (different) absolute frequencies correspond 
to the exact same encounter frequency when $\omega_{e}<\frac{1}{4 \psi}$, resulting in the 3-to-1 mapping between a corresponding set of frequencies. ${ }^{1}$ In Figure 2, the set of absolute frequencies, leading to the same encounter frequency when $\omega_{e}<\frac{1}{4 \psi}$, has been included below the plot area. As a consequence of the 3-to-1 mapping for a ship advancing in following waves, spectrum transformation from the one domain to the other is not straight-forward.

To have a more complete view of the transformation problem, it is worthwhile to consider the relationship in Eq. (2) together with the requirement imposing energy equivalence, Eq. (1). The conservation of energy leads to the following expressions dependent on the "direction" of transformation:

$$
\begin{aligned}
& S_{0}\left(\omega_{0}\right)=S_{e}\left(\omega_{e}\right) \frac{d \omega_{e}}{d \omega_{0}} \\
& S_{e}\left(\omega_{e}\right)=S_{0}\left(\omega_{0}\right) \frac{d \omega_{0}}{d \omega_{e}}
\end{aligned}
$$

and it is seen that different derivatives are needed. In the former case (Eq. (3), from encounter to absolute domain, the derivative is immediately obtained from Eq. (2); i.e.

$$
\frac{d \omega_{e}}{d \omega_{0}}=1-2 \omega_{0} \psi
$$

Obviously, the reciprocal derivative $\frac{d \omega_{0}}{d \omega_{e}}$, needed in Eq. (4), can be formed by taking the inverse of Eq. (5). Thus, the transformation formulas read:

$$
\begin{aligned}
& S_{0}\left(\omega_{0}\right)=S_{e}\left(\omega_{e}\right)\left(1-2 \omega_{0} \psi\right) \\
& S_{e}\left(\omega_{e}\right)=S_{0}\left(\omega_{0}\right) \frac{1}{1-2 \omega_{0} \psi}
\end{aligned}
$$

It is important to emphasise that the formulas, in either case, strictly require the absolute frequency to be known, and this will not initially be the case when (time-domain) measurements in the encounter domain are in study. For theoretical spectral calculations, on the other hand, the absolute (wave) frequency is typically specified as the very initialisation of the numerical calculations. In this situation, the transformation formulas can be directly applied, and an example is considered in Figure 3. Here, the Bretschneider spectrum $\left(H_{s}=3.0 \mathrm{~m}, T_{z}=8.0 \mathrm{~s}\right)$, previously studied in Figure 1, is shown when it

\footnotetext{
${ }^{1}$ While it may be a 3 -to-1 mapping in the one direction, it will be a 1-to-3 mapping in the other. Anyhow, the problem will generally from this point just be referred to as a 3-to-1 mapping problem.
} 
is transformed from the absolute domain to an encountered domain observed on a ship advancing with speed $U=10$ knots in following sea at heading $\beta=30$ deg. relative to the waves.

Indeed, Figure 3 shows how the encountered wave spectrum is 'distorted' in following waves, where three points are noteworthy: (1) the occurrence of negative spectral ordinates, (2) multiple spectral ordinates at some encounter frequencies, (3) the singularity at $\omega_{0}=\frac{1}{2 \psi}$. The observation (2) of multiple values at some frequencies is because of the frequency mapping using Eq. (2) that results in a non-ordered set of encounter frequencies for an ordered set of absolute frequencies (lowest to highest). Consequently, the difference term $d \omega_{e}$ will not only attain positive but also negative values for a corresponding $\omega_{e}$-vector. The observation of negative spectral ordinates is thus related to the non-ordered set of encounter frequencies; emphasising that a negative wave spectral ordinate - in the encounter domain - is not equivalent to the wave energy being negative. This is so exactly because the frequency derivative, $\frac{d \omega_{e}}{d \omega_{0}}$, will also be negative, so that energy itself is positive; since energy is the product of the spectral ordinate and the frequency derivative. Altogether, the (encounter) spectrum shown in Fig. 3 is 'mathematically consistent' and the spectrum can be used directly when/if transformation (back) to the absolute domain is carried out by use of Eqs. (3) and (6); but, it is stressed that a transformation is valid only for the initial pre-specified (and ordered) set of absolute frequencies $\omega_{0}$ with a specific, but non-ordered, corresponding set of encounter frequencies

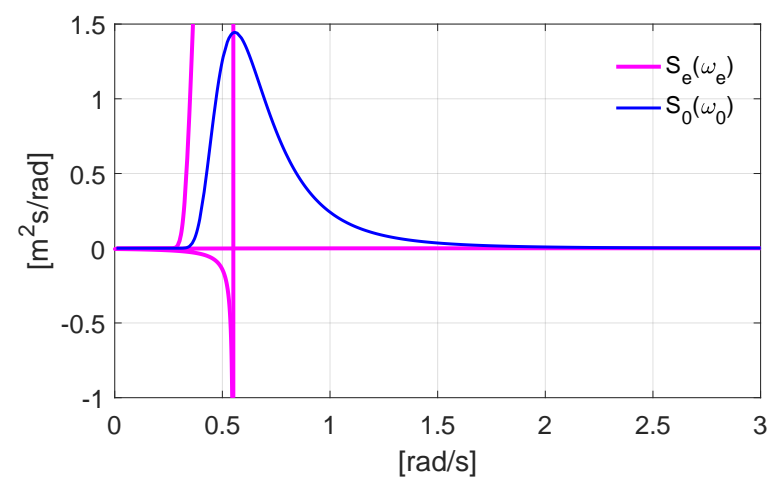

Figure 3: Transformation of spectrum in following sea (Bretschneider spectrum with $H_{s}=3.0 \mathrm{~m}$, $T_{z}=8.0 \mathrm{~s}$ ) leads to a heavily distorted encounter spectrum. The vessel speed is 10 knots and heading is $30 \mathrm{deg}$. 


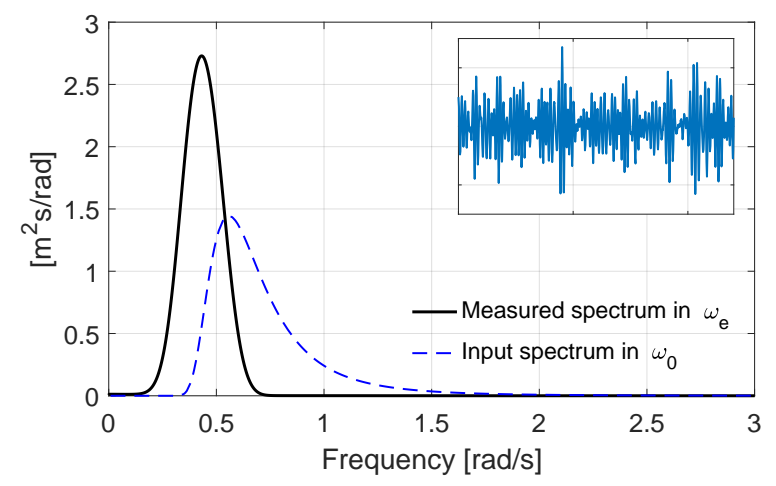

Figure 4: A measured (i.e. simulated) wave elevation record (smaller plot in right corner) transforms to an energy spectrum having only positive ordinates at a strictly ordered set of frequencies.

$\omega_{e}$.

The situation is quite different when real time history measurements are observed and, as a postprocess, analysed by spectral calculations. In this case, the set of (observed) encounter frequencies will be strictly ordered (lowest to highest) and, as result, the encounter spectral ordinates take only positive values. This is illustrated in Figure 4 that shows the encountered wave spectrum observed from a wave elevation record measured (i.e. simulated) on the advancing vessel studied previously in Figure 3; i.e. $U=10$ knots in following waves at a heading $\beta=30 \mathrm{deg}$., and $H_{s}=3.0 \mathrm{~m}, T_{z}=8.0 \mathrm{~s}$. The spectrum is obtained by use of fast Fourier transformation (FFT) with smoothing. ${ }^{2}$ The implication by having the encounter spectrum for the ordered set of encounter frequencies $\omega_{e}$ is that Eq. (6) cannot be (immediately) applied to transform the spectrum to the absolute domain.

The fundamental problem(s) and associated contemplations addressed above are summarised and illustrated in Figure 5 that applies to following waves, when Eqs. (1) and (2), including Figures 1 and 2, are combined. Basically, Figure 5 illustrates how confined energy "lumps" in the one domain are mapped into the other domain. In this study, the interest concerns the mapping of energy from encounter to absolute domain. Particularly, it is important to evaluate the situation when $\omega_{e}<\frac{1}{4 \psi}$, cf. Figure 2, since, in this situation, one encounter-wave component will be mapped into three absolute-wave com-

\footnotetext{
${ }^{2}$ In the present example, the built-in function cpsd in MATLAB ${ }^{\circledR}$ is used.
} 
ponents. Thus, from Figure 5 it becomes evident that, generally, it cannot be uniquely determined how to distribute the energy lump $A_{e 1}$ into the three lumps $A_{\omega 1}, A_{\omega 2}$, and $A_{\omega 4}$. Importantly, however, it is vital to note that, although there is no unique solution

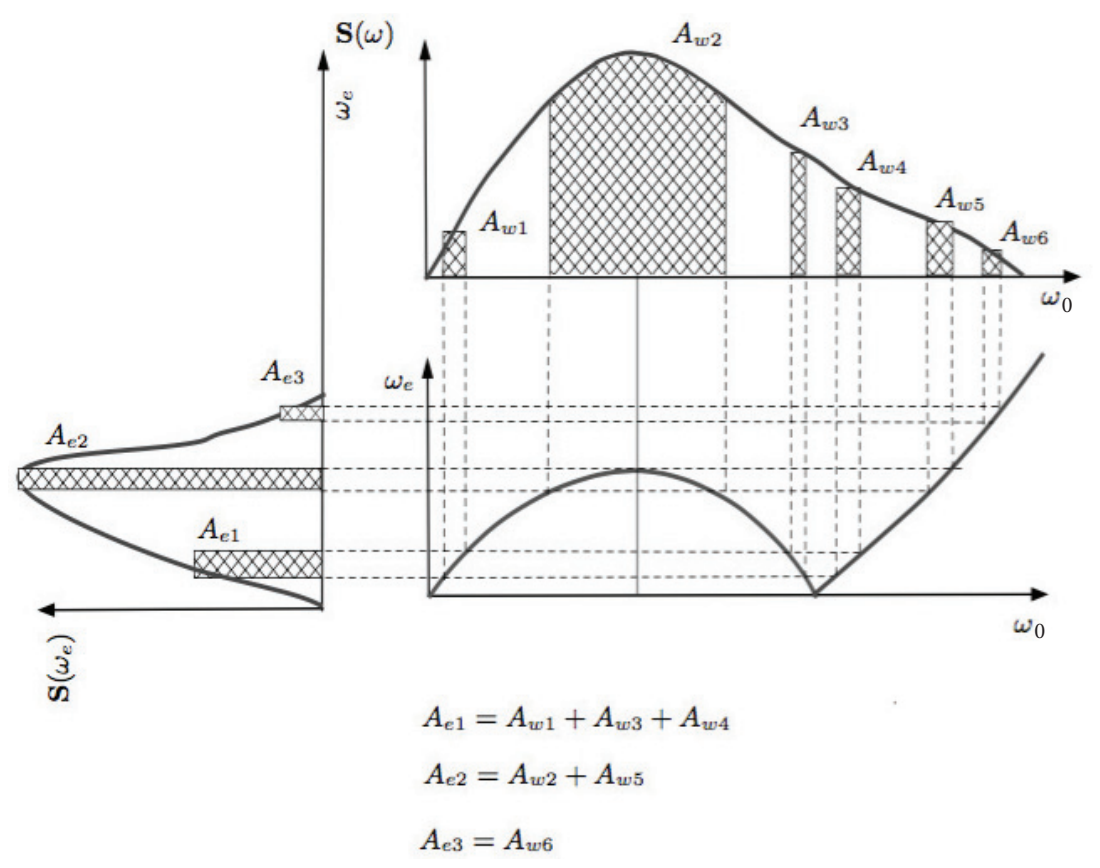

Figure 5: Transformation of wave spectrum. Appears in [? ].

on how to actually divide the energy lump $A_{e 1}$ itself into three corresponding lumps $A_{\omega 1}, A_{\omega 3}$, and $A_{\omega 4}$, it is exactly known at which specific frequencies $\omega_{01}, \omega_{03}$, and $\omega_{04}$, the lumps need to be assigned. The frequencies follow directly from Figure 2 and are repeated here:

$$
\begin{array}{ll}
\omega_{01}=\frac{1-\sqrt{1-4 \psi \omega_{e 1}}}{2 \psi} & \text { (Region I, Fig. 2) } \\
\omega_{03}=\frac{1+\sqrt{1-4 \psi \omega_{e 1}}}{2 \psi} & \text { (Region II, Fig. 2) } \\
\omega_{04}=\frac{1+\sqrt{1+4 \psi \omega_{e 1}}}{2 \psi} & \text { (Region III, Fig. 2) }
\end{array}
$$

Obviously, this knowledge can be used to infer about how to distribute the energy from encounter to absolute domain. Thus, as a (first) try, Eqs. (6) and (8)-(10) can be used somewhat "blindly" to transform from encounter to absolute domain at a set of absolute, 


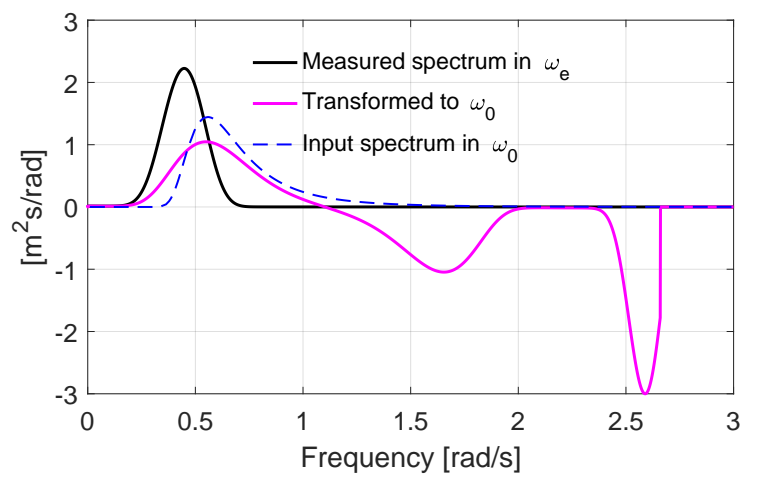

Figure 6: Direct transformation of an encounter wave spectrum - measured in following sea - may leads to a wrong absolute-spectrum in the $\omega_{0}$-domain.

but sorted, frequencies $\omega_{0}$. This has been done for the situation studied previously in Figure 4. However, an erroneous result is achieved; clearly illustrated in Figure 6 that reflects evidently the problem imposed because of the 3 -to-1 mapping between a corresponding set of frequencies. Part of the concern in the transformation is related to the illustration in Figure 5 since the illustration may mislead if transformation is tried out directly as indicated in the illustration. Instead, it is strictly necessary to include an intermediate stage in the transformation process, where the distorted, but mathematically consistent, version of the encounter wave spectrum is introduced; keeping in mind that this spectrum has not only positive values but also negative values at some frequencies, cf. Figure 3.

Altogether, the considerations and discussions in this section should have shed light on the practical complications when an encounter (wave) spectrum is transformed into a corresponding spectrum in the absolute domain. At the same time, the section should have indicated a viable approach from which to make the actual transformation. Hence, the next section suggests a procedure on how the transformation problem can be practically dealt with, and two pseudo algorithms will be explicitly outlined.

\section{Algorithms transforming from encounter to absolute domain}

As part of the set-up of the algorithms to transform from encounter to absolute domain the following will be assumed: 
- Vessel forward-speed $U>0$ and (mean) heading $\beta$ relative to the wave system are known.

- The observed wave system, from the advancing ship, is described by the encounter wave spectrum $S_{e}\left(\omega_{e}\right)$ that has been estimated by some means. The spectrum is discretised as $S_{i}^{e n c}=S_{e}\left(\omega_{e, i}\right)$, where $\omega_{e, i}$ is the i-th element of a vector containing the set of encounter frequencies. The number of elements in the vector is $N_{e}$, and $\omega_{e, i}<\omega_{e, i+1}$ for any $i$.

- The n-th order spectral moment, in the encounter domain, can be calculated from the encounter spectrum and, subsequently, estimates of integrated sea state parameters such as significant wave height and zero-upcrossing period are obtained:

$$
\begin{aligned}
& m_{n}=\int_{0}^{\infty} \omega_{e}^{n} S_{e}\left(\omega_{e}\right) d \omega_{e} \\
& H_{s}=4 \sqrt{m_{0}}, \quad T_{z}^{e n c}=2 \pi \sqrt{\frac{m_{0}}{m_{2}}}
\end{aligned}
$$

It is important to realise that any characteristic wave period, e.g. zero-upcrossing period as derived from the spectral moments, applies to the encounter domain exclusively. This is, however, not the case for the significant wave height, since this number is a measure of the wave system's total energy that preserves no matter the domain.

- Some theoretical and parameterised wave spectrum $S_{p}\left(\omega_{0}\right)$ (e.g., Bretschneider, JONSWAP) must be available, so that a spectral ordinate can be calculated at any absolute frequency $\omega_{0}$ with given sea state parameters.

Based on the assumptions listed above, two pseudo algorithms to transform accordingly are given in the scripts seen in Algorithms 1 and 2, where focus in both cases is merely on cases in following waves $\beta=[0-90[\mathrm{deg}$.

\subsection{Algorithm 1}

The script seen in Algorithm 1, can be supplemented with some additional comments:

Line 4 assigns the discrete set of an encounter frequency $\hat{\omega}_{i}$ and a corresponding wave spectrum ordinate. The spectrum ordinate is taken as the estimate/measurement denoted by $\hat{S}_{e, i}$, or $\hat{S}_{0, i}$ for the transformed one. 


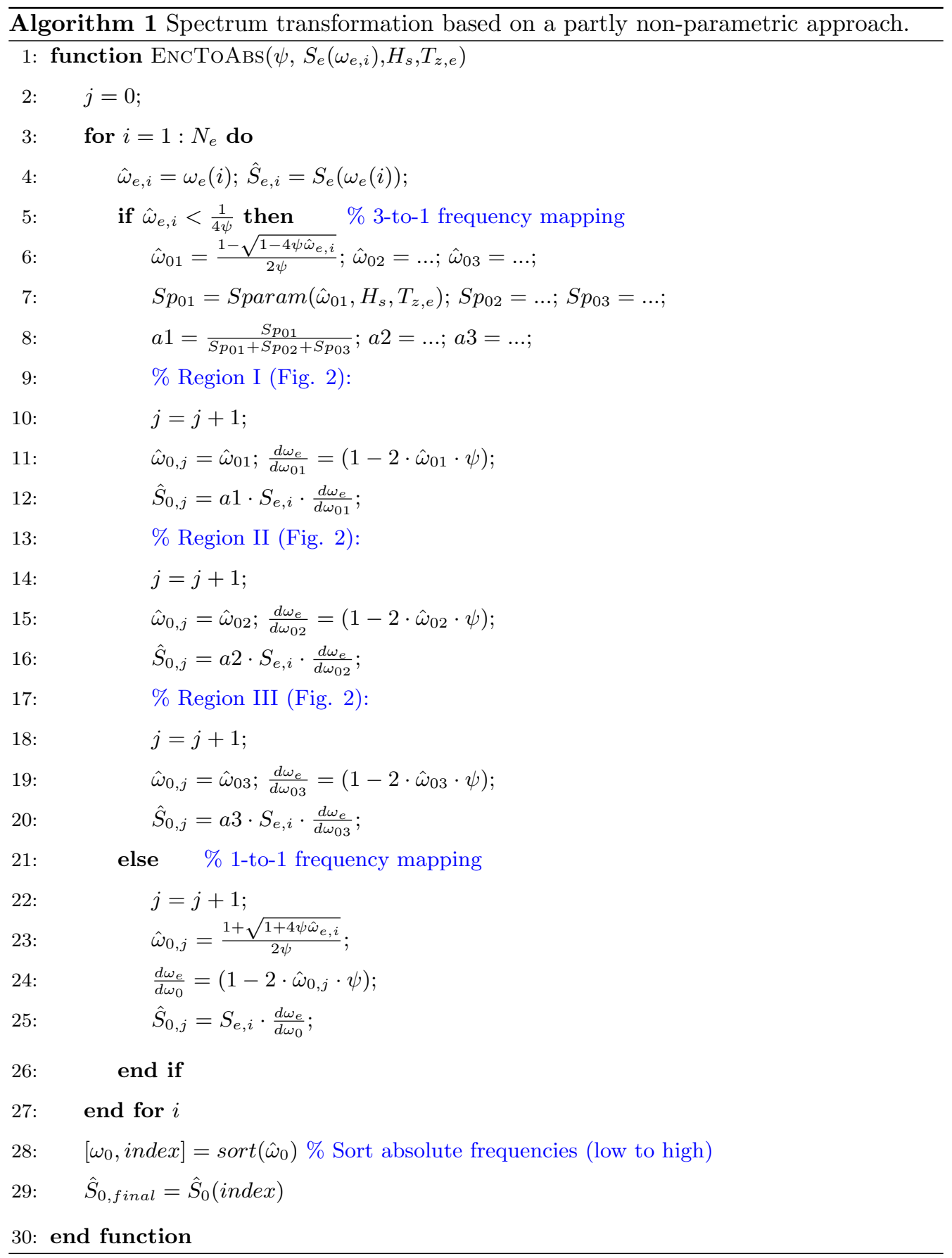


Line 5 The transformation must be divided into two parts by an if-else loop separated by the singularity at $\omega_{e}=\frac{1}{4 \psi}$.

Line 6 provides the set of absolute frequencies calculated in accordance with Eqs. (8)-(9).

Line 7 introduces a theoretical and parameterised wave spectrum $S_{p}$ dependent on absolute frequency $\omega_{0}$. Input parameters for the parameterised spectrum will, typically, be significant wave height and a characteristic wave period. The significant wave height is directly obtained from the encounter-wave spectrum, but this is not possible for the period. Further remarks about the "selection" of the characteristic wave period will be given later.

Line 8 provides three 'scaling ratios' $\left\{a_{1}, a_{2}, a_{3}\right\}$ based on the parametric wave spectrum.

Lines 9-20 Any one estimated spectral ordinate $\hat{S}_{e, i}$, in encounter domain, needs to be "simultaneously" transformed into three ordinates in the (true) absolute domain; $\hat{S}_{01, i}, \hat{S}_{02, i}$, and $\hat{S}_{03, i}$, where (only) the frequencies $\left\{\omega_{01}, \omega_{02}, \omega_{03}\right\}$ are known. However, for a given parameterised spectrum, $S_{p}\left(\omega_{0} \mid H_{s}, T_{z}, \ldots\right)$, a known frequency implies immediately a known spectral ordinate, if integrated wave parameters $\left(H_{s}, T_{z}, \ldots\right)$ are also available. Now, from the 'parameterised' ordinates $S p_{01}=S_{p}\left(\omega_{01}\right), S p_{02}=S_{p}\left(\omega_{02}\right)$, $S p_{03}=S_{p}\left(\omega_{03}\right)$, three ratios are formed

$$
\begin{aligned}
& a_{1}=\frac{\left.S_{p 01} d \omega_{0}\right|_{\omega 01}}{\left.S_{p 01} d \omega_{0}\right|_{\omega 01}+\left.S_{p 02} d \omega_{0}\right|_{\omega 02}+\left.S_{p 03} d \omega_{0}\right|_{\omega 03}} \\
& a_{2}=\frac{\left.S_{p 02} d \omega_{0}\right|_{\omega 02}}{\left.S_{p 01} d \omega_{0}\right|_{\omega 01}+\left.S_{p 02} d \omega_{0}\right|_{\omega 02}+\left.S_{p 03} d \omega_{0}\right|_{\omega 03}}\left.S_{p 03} d \omega_{0}\right|_{\omega 03} \\
& a_{3}=\frac{\left.S_{p 02} d \omega_{0}\right|_{\omega 02}+\left.S_{p 03} d \omega_{0}\right|_{\omega 03}}{\left.S_{p 01} d \omega_{0}\right|_{\omega 01}+S_{00}}
\end{aligned}
$$

where $\left.\ldots\right|_{\omega}$ indicates that the expression is evaluated at frequency $\omega$. These ratios are relative measures on how energy is distributed with frequency for the parameterised spectrum and, as the central point, the same relative energy distribution is then applied to the estimated spectrum in the absolute domain. Hence,

$$
\begin{gathered}
\frac{\left.\hat{S}_{01} d \omega_{0}\right|_{\omega 01}}{\left.\hat{S}_{01} d \omega_{0}\right|_{\omega 01}+\left.\hat{S}_{02} d \omega_{0}\right|_{\omega 02}+\left.\hat{S}_{03} d \omega_{0}\right|_{\omega 03}} \\
\frac{\left.\hat{S}_{02} d \omega_{0}\right|_{\omega 02}}{\left.\hat{S}_{01} d \omega_{0}\right|_{\omega 01}+\left.\hat{S}_{02} d \omega_{0}\right|_{\omega 02}+\left.\hat{S}_{03} d \omega_{0}\right|_{\omega 03}}
\end{gathered} a_{14}
$$




$$
\frac{\left.\hat{S}_{03} d \omega_{0}\right|_{\omega 03}}{\left.\hat{S}_{01} d \omega_{0}\right|_{\omega 01}+\left.\hat{S}_{02} d \omega_{0}\right|_{\omega 02}+\left.\hat{S}_{03} d \omega_{0}\right|_{\omega 03}}=a_{3}
$$

In Eqs. (16)-(18), it is important to realise that the individual components $\left\{\hat{S}_{01}, \hat{S}_{02}\right.$, $\left.\hat{S}_{03}\right\}$ are unknown, but the sum of them is not, since

$$
\left.\hat{S}_{01} d \omega_{0}\right|_{\omega 01}+\left.\hat{S}_{02} d \omega_{0}\right|_{\omega 02}+\left.\hat{S}_{03} d \omega_{0}\right|_{\omega 03}=\hat{S}_{e} d \omega_{e}
$$

Consequently, the individual spectral ordinates, in the absolute domain, can be calculated as

$$
\begin{aligned}
& \hat{S}_{01}=\left.a_{1} \cdot \hat{S}_{e} \frac{d \omega_{e}}{d \omega_{0}}\right|_{\omega_{01}} \quad \text { (Region I, Fig. 2) } \\
& \hat{S}_{02}=-\left.a_{2} \cdot \hat{S}_{e} \frac{d \omega_{e}}{d \omega_{0}}\right|_{\omega_{02}} \quad \text { (Region II, Fig. 2) } \\
& \hat{S}_{03}=-\left.a_{3} \cdot \hat{S}_{e} \frac{d \omega_{e}}{d \omega_{0}}\right|_{\omega_{03}} \quad \text { (Region III, Fig. 2) }
\end{aligned}
$$

taking note that the derivatives in Regions II and III are negative, and, hence, the mathematically consistent spectrum, having negative values in those regions, must be introduced.

Lines 28-29 The mapped frequencies $\omega_{0}$ in the absolute domain (Line 6) will be unsorted. Therefore, sorting from low to high is conducted, and the spectral ordinates are sorted accordingly. Note also that the number of elements in $\hat{S}_{0, \text { final }}$ will be different (larger) than in the corresponding vector $\hat{S}_{e}$ that has $N_{e}$ elements.

\subsection{Algorithm 2}

While Algorithm 1 produces a transformed absolute spectrum which is (partly) nonparametric, the other transformation procedure, see Algorithm 2, is entirely parametric and is based on an optimisation of a set of wave parameters. Thus, Algorithm 2 assumes the (absolute) wave spectrum to have the shape of a general, parameterised wave spectrum, dependent on a set of characteristic wave parameters. The algorithm should be read with the following supplementary remarks; take note that some remarks will be repetitions from Algorithm 1:

Line 3 The optimisation procedure must be initialised with a starting guess on the set of wave parameters that is optimised. The specific pseudo algorithm considers a parameterised unimodal wave spectrum with only two wave parameters $\left(H_{s}, T_{z}\right)$, but obviously a more general spectrum can easily be implemented instead. 


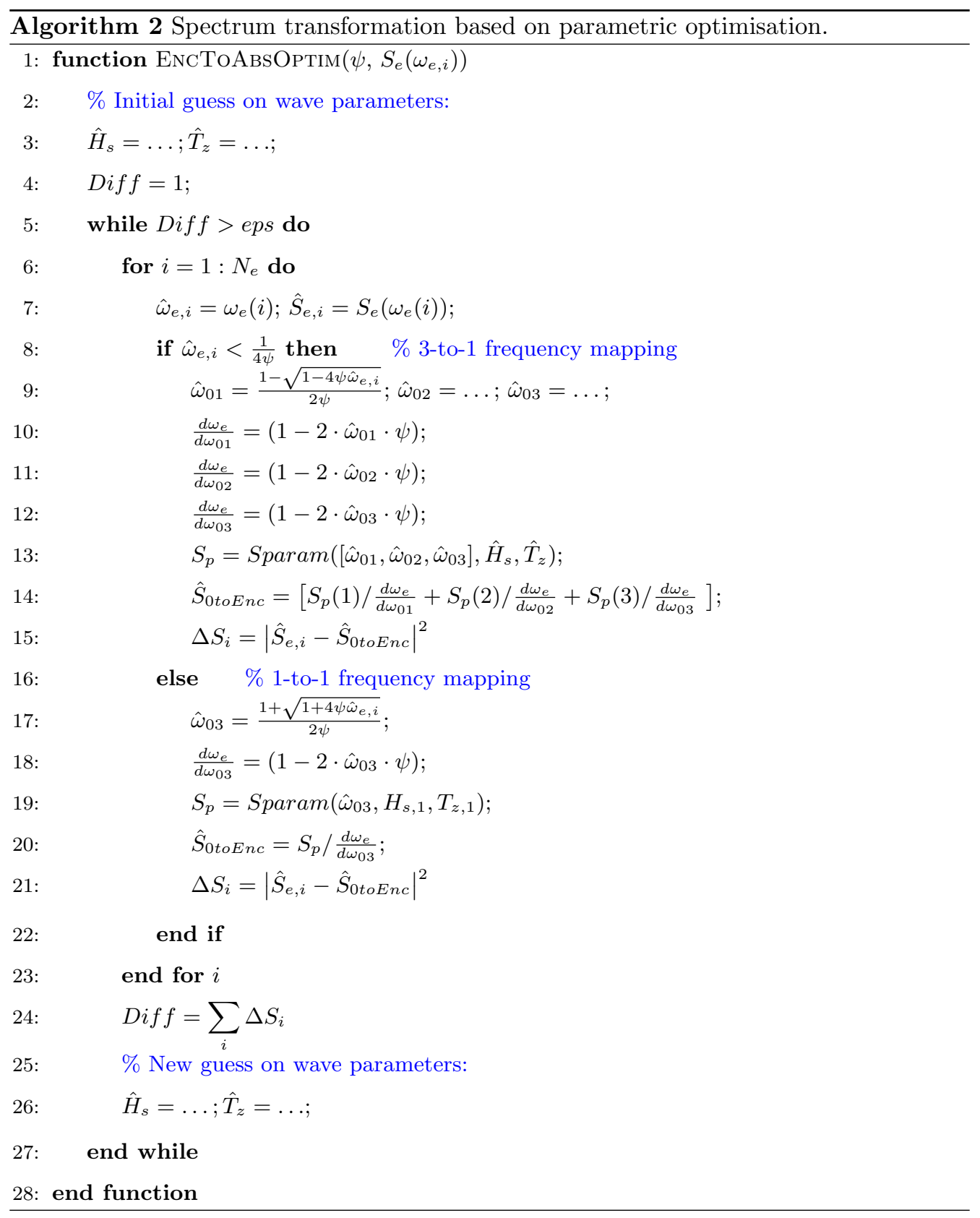


Line 4 initialises the while loop that controls the optimisation.

Line 7 assigns the discrete set of an encounter frequency $\hat{\omega}_{i}$ and a corresponding wave spectrum ordinate. The spectrum ordinate is taken as the estimate/measurement denoted by $\hat{S}_{e, i}$, or $\hat{S} 0, i$ for the transformed one in the absolute domain.

Line 8 The optimisation must be split in two parts controlled by an if-else loop: In the first part, $\omega_{e}<\frac{1}{4 \psi}$ which means that three 'energy lumps' from the absolute domain contribute to the one lump in the (measured) encounter domain, cf. Figure 5.

Lines 9-12 transforms the encounter frequency into three corresponding absolute frequencies, and subsequently the derivatives are obtained.

Line 13 The three absolute frequencies map into three spectral ordinates of a given parameterised wave spectrum. The ordinates are stored as a vector with three elements, and apply to the absolute domain.

Line 14 transforms to encounter domain using the reciprocal value of the deriva$\operatorname{tive}(\mathrm{s})$.

Line 15 calculates the square of the error between the (measured) encounter wave spectrum ordinate and the corresponding transformed absolute one for the particular encounter frequency (line 7) in study.

Lines 16-22 is the second part of the if-else loop which considers the case when $\omega_{e}>\frac{1}{4 \psi}$, where the frequency mapping is 1 -to- 1 .

Line 24 calculates the sum of the squared errors and, hence, a new guess can be made on the wave parameters to be optimised.

The proposed pseudo algorithm sketches schematically the solution procedure. However, it should be realised that the optimisation of the set of wave parameters can be made more elaborate than the rather brute-force approach outlined in Algorithm 2. This can be achieved by formulating a cost-function which is minimised by applying, for instance, a nonlinear programming solver; maybe taking into account also some mathematical and/or physical constraints related to the problem.

In the next section, several case studies are considered where the main and sole objective is to transform a wave energy spectrum from encounter domain to absolute domain. The actual data is composed by numerical simulations of time history recordings of an encountered wave elevation reading, observed from an advancing ship. This setup allows 
for a realistic setting where the encounter-wave spectrum is estimated on the advancing vessel and, therefore, needs to be transformed to the absolute domain. Algorithms 1 and 2 have both been applied to the data but the focus will be exclusively on the results obtained by Algorithm 1. Generally, reasonable results have been achieved also by Algorithm 2 but the procedure turned out to be somewhat sensitive to the initial guess of wave parameters (Line 3). It is therefore decided to leave this particular algorithm for some further work before it is mature for practical engineering cases.

\section{Case studies: Application to simulated data}

In the particular context a realistic scenario is the following: A ship navigates in an irregular seaway. The vessel advances with given forward speed $U$ and heading $\beta$ relative to the seaway; $\beta=180 \mathrm{deg}$. is head sea. The ship has installed a sea state estimator that estimates, one way or the other, the encountered wave system given in terms of a wave energy spectrum. From the encounter spectrum, significant wave height $H_{s}$ and any characteristic period, say, the 'encounter zero-upcrossing period' $T_{z, e}$, are immediately available and, moreover, an estimate of the relative heading between waves and vessel is included. This particular set of wave measurements is the outcome of the wave buoy analogy when a specific implementation of it is introduced [? ]. In the scenarios studied later, however, the actual encounter-wave spectrum is derived by postprocessing time series data of numerically simulated wave elevations based on a set of known wave parameters.

For real-case data, uncertainty is (or must be) associated to the estimate of the wave system; not to mention the uncertainty related to the forward speed-through-water of the vessel [? ]. Anyhow, in this study considering numerical simulations, the uncertainties of operational and environmental parameters will be neglected, since the uncertainties themselves are of no importance for the actual transformation procedure/process. In the same line, the simulations of the wave elevation records are made without any artificial noise. Altogether, this makes it possible to exclusively test the performance of the transformation algorithm(s), without disturbance from any 'unknown factors'. 


\subsection{Wave scenarios}

Various test cases form the background for the evaluation of the performance of Algorithm 1. Each test case is represented by a certain long-crested input-wave system characterised by a parameterised wave energy spectrum and its associated (true) integrated wave parameters. This input-wave system is formulated/given in the absolute domain. In order to simulate the scenario described above, advance speed and heading relative to the wave system are specified and, thus, the encountered wave elevation record in the time domain can be easily generated for a ship advancing in the particular wave system. In this study, standard time-domain simulation, cf. subsection 4.2, is used to generate the time series of the wave elevation, but taking note that two corresponding time histories are generated; (i) the wave elevation record observed from a fixed point relative to the frame of reference of the considered wave system, and (ii) the wave record measured/encountered at an advancing ship. In either case, fast Fourier transformation (FFT) is subsequently applied to obtain the measured wave spectrum; emphasising that the one spectrum, corresponding to (i), will be a function of the absolute frequency $\omega_{0}$, whereas the other spectrum, that of (ii), will be a function of the encounter frequency $\omega_{e}$. The central task for any transformation algorithm is therefore to convert the measured encounter-wave spectrum into the absolute domain. The transformed spectrum, from (ii), can thus be directly compared to the absolute wave spectrum from (i).

An overview of the test cases is seen in Tables 1 and 2 that specify the (absolute) input-wave parameters. Based on the parameters of a particular test case, $2 \times 20$ sets of corresponding time history elevation records are obtained; one set belonging to the advancing ship and one set for a non-moving observer. The need for several records, here 20 , is due to the fact that a statistical evaluation of the algorithm's performance is necessary, since a single, finite time history recording is just one out of the infinitely many that comprise the "complete" wave system. The individual wave elevation records are 2 hours long and made from 1,000 wave components spaced non-equidistantly, see also subsection 4.2 .

The wave scenarios represented by the parameters in Table 1 are described by a Bretschneider wave spectrum, 'B', or a JONSWAP spectrum, 'J'. That is, for the generation of the wave elevation records, the input spectrum, equivalently the generating 
Table 1: Summary of test cases using a unimodal wave spectrum as the "generating spectrum"; it being either of Bretschneider type ('B') or JONSWAP type ('J'), where the latter has $\gamma=2.0$ in every case.

\begin{tabular}{|c|c|c|c|c|c|c|}
\hline Case & Type & $U[$ knots $]$ & $\beta\left[^{\circ}\right]$ & $T_{z}[\mathrm{~s}]$ & $H_{s}[\mathrm{~m}]$ & $\gamma[-]$ \\
\hline $\mathrm{A} 1 / \mathrm{G} 1$ & 'B'/'J' & 10.0 & 0 & 6.0 & 3.0 & $-/ 2.0$ \\
\hline $\mathrm{B} 1 / \mathrm{H} 1$ & 'B'/'J' & 15.0 & 0 & 6.0 & 3.0 & $-/ 2.0$ \\
\hline $\mathrm{C} 1 / \mathrm{I} 1$ & 'B'/'J' & 20.0 & 0 & 6.0 & 3.0 & $-/ 2.0$ \\
\hline $\mathrm{A} 2 / \mathrm{G} 2$ & 'B'/'J' & 10.0 & 30 & 6.0 & 3.0 & $-/ 2.0$ \\
\hline $\mathrm{B} 2 / \mathrm{H} 2$ & 'B'/'J' & 15.0 & 30 & 6.0 & 3.0 & $-/ 2.0$ \\
\hline $\mathrm{C} 2 / \mathrm{I} 2$ & 'B'/'J' & 20.0 & 30 & 6.0 & 3.0 & $-/ 2.0$ \\
\hline A3/G3 & B'/'J' & 10.0 & 60 & 6.0 & 3.0 & $-/ 2.0$ \\
\hline B3/H3 & B'/'J' & 15.0 & 60 & 6.0 & 3.0 & $-/ 2.0$ \\
\hline C3/I3 & B'/'J' & 20.0 & 60 & 6.0 & 3.0 & $-/ 2.0$ \\
\hline $\mathrm{A} 4 / \mathrm{G} 4$ & B'/'J' & 10.0 & 0 & 8.0 & 3.0 & $-/ 2.0$ \\
\hline $\mathrm{B} 4 / \mathrm{H} 4$ & B'/'J' & 15.0 & 0 & 8.0 & 3.0 & $-/ 2.0$ \\
\hline $\mathrm{C} 4 / \mathrm{I} 4$ & B'/'J' & 20.0 & 0 & 8.0 & 3.0 & $-/ 2.0$ \\
\hline A5/G5 & B'/'J' & 10.0 & 30 & 8.0 & 3.0 & $-/ 2.0$ \\
\hline $\mathrm{B} 5 / \mathrm{H} 5$ & B'/'J' & 15.0 & 30 & 8.0 & 3.0 & $-/ 2.0$ \\
\hline C5/I5 & B'/'J' & 20.0 & 30 & 8.0 & 3.0 & $-/ 2.0$ \\
\hline A6/G6 & B'/'J' & 10.0 & 60 & 8.0 & 3.0 & $-/ 2.0$ \\
\hline $\mathrm{B} 6 / \mathrm{H} 6$ & B'/'J' & 15.0 & 60 & 8.0 & 3.0 & $-/ 2.0$ \\
\hline C6/I6 & B'/'J' & 20.0 & 60 & 8.0 & 3.0 & $-/ 2.0$ \\
\hline $\mathrm{A} 7 / \mathrm{G} 7$ & B'/'J' & 10.0 & 0 & 10.0 & 3.0 & $-/ 2.0$ \\
\hline $\mathrm{B} 7 / \mathrm{H} 7$ & B'/'J' & 15.0 & 0 & 10.0 & 3.0 & $-/ 2.0$ \\
\hline $\mathrm{C} 7 / \mathrm{I} 7$ & $\mathrm{~B}^{\prime} /{ }^{\prime} \mathrm{J} '$ & 20.0 & 0 & 10.0 & 3.0 & $-/ 2.0$ \\
\hline A8/G8 & B'/'J' & 10.0 & 30 & 10.0 & 3.0 & $-/ 2.0$ \\
\hline $\mathrm{B} 8 / \mathrm{H} 8$ & B'/'J' & 15.0 & 30 & 10.0 & 3.0 & $-/ 2.0$ \\
\hline C8/I8 & B'/'J' & 20.0 & 30 & 10.0 & 3.0 & $-/ 2.0$ \\
\hline $\mathrm{A} 9 / \mathrm{G} 9$ & B'/'J' & 10.0 & 60 & 10.0 & 3.0 & $-/ 2.0$ \\
\hline B9/H9 & B'/'J' & 15.0 & 60 & 10.0 & 3.0 & $-/ 2.0$ \\
\hline C9/I9 & B'/'J' & 20.0 & 60 & 10.0 & 3.0 & $-/ 2.0$ \\
\hline
\end{tabular}

spectrum, is either taken as the Bretschneider or the JONSWAP spectrum. The mathematical formulations of the two spectra are seen in Eqs. (23) and (24).

$$
\text { Bretschneider: } \quad S=173 \frac{H_{s}^{2}}{T^{4} \omega_{0}^{5}} \exp \left[-\frac{692}{T^{4} \omega_{0}^{4}}\right]
$$

where the characteristic period $T$ depends on which statistical period is given. The following substitutions apply: $T=T_{1}$ for the mean period $T_{1}, T=0.772 T_{p}$ for the peak period $T_{p}$, or by $T=1.086 T_{z}$ for the zero-upcrossing period $T_{z}$.

$$
\text { JONSWAP: } \quad S=320 \frac{H_{s}^{2}}{T^{4} \omega_{0}^{5}} \exp \left[-\frac{1950}{T^{4} \omega_{0}^{4}}\right] \gamma^{J} ; J=\exp \left[-\left(\frac{\frac{\omega_{0}}{\omega_{p}}-1}{\sigma \sqrt{2}}\right)^{2}\right]
$$


Table 2: Summary of test cases using a bimodal (two-peaked) wave spectrum as the generating spectrum.

The two input spectra are in all cases of the Bretschneider type.

\begin{tabular}{lcccc}
\hline Case & $U[\mathrm{knots}]$ & $\beta\left[^{\circ}\right]$ & $T_{z}[\mathrm{~s}]$ & $H_{s}[\mathrm{~m}]$ \\
\hline D1 & 10.0 & 0 & $8.0+13.0$ & $3.0+2.0$ \\
E1 & 15.0 & 0 & $8.0+13.0$ & $3.0+2.0$ \\
F1 & 20.0 & 0 & $8.0+13.0$ & $3.0+2.0$ \\
D2 & 10.0 & 30 & $8.0+13.0$ & $3.0+2.0$ \\
E2 & 15.0 & 30 & $8.0+13.0$ & $3.0+2.0$ \\
F2 & 20.0 & 30 & $8.0+13.0$ & $3.0+2.0$ \\
D3 & 10.0 & 60 & $8.0+13.0$ & $3.0+2.0$ \\
E3 & 15.0 & 60 & $8.0+13.0$ & $3.0+2.0$ \\
F3 & 20.0 & 60 & $8.0+13.0$ & $3.0+2.0$ \\
\hline
\end{tabular}

where the characteristic period $T$ depends on which statistical period is given. The following substitutions apply: $T=1.199 T_{1}$ for the mean period $T_{1}, T=T_{p}$ for the peak period $T_{p}$, or by $T=1.287 T_{z}$ for the zero-upcrossing period $T_{z}$. The peakedness factor is given by $\gamma$, while the step function $\sigma$ takes values as $\sigma=0.07$ for $\omega_{0}<\omega_{p}$, and $\sigma=0.09$ for $\omega_{0}>\omega_{p}$ with $\omega_{p}=\frac{2 \pi}{T_{p}}$. Note that the absolute frequency $\omega_{0}$ is the independent variable to either spectrum.

Table 1 represents unimodal (i.e. single peaked) sea states, whereas Table 2 shows cases corresponding to bimodal (i.e. double peaked) sea states which, in general, are combinations of a wind sea system and swells occurring at the same time.

Examples of the generating wave spectra are seen in Figure 7, which applies to some of

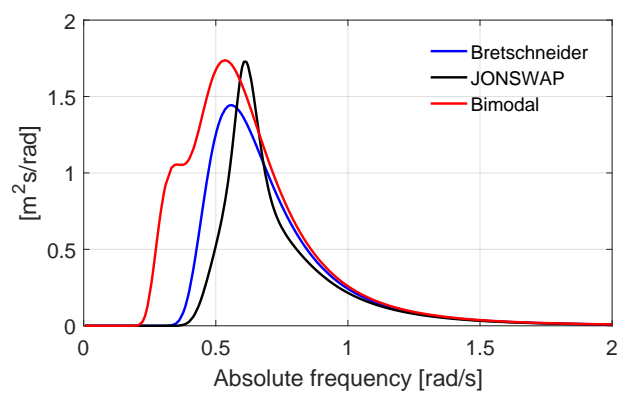

Figure 7: Examples of the generating spectra of Cases A-C, D-F, and G-I using, respectively, a Bretschneider, a JONSWAP, or the summation of two Bretschneider spectra ('Bimodal'). The plots apply for the following wave parameters: Bretschneider, $H_{s}=3.0 \mathrm{~m}, T_{z}=8.0 \mathrm{~s}$; JONSWAP, $H_{s}=3.0 \mathrm{~m}$, $T_{z}=8.0 \mathrm{~s}, \gamma=2.0$, Bimodal, $H_{s}=\{3.0,2.0\} \mathrm{m}, T_{z}=\{8.0,13.0\} \mathrm{s}$. 
the cases considered in Tables 1 and 2 . The next subsection outlines briefly how the time histories of the wave elevation are generated using the input parameters from Tables 1 and 2. Subsequently, the spectrum transformations are made on the wave spectra obtained from the actual time histories, where the specific spectra will be distorted versions of the generating spectra that may be similar to those seen in Figure 7.

\subsection{Time history generation}

The wave elevation is assumed to have properties from a Gaussian process, and thus the time history record at a single point can be generated, e.g. [? ], using a set of uncorrelated, standard normal distributed variables $u_{n}$ and $\bar{u}_{n}$. Hence,

$$
\eta(t)=\sum_{n=1}^{N_{0}}\left[u_{n} c_{n}(t)+\bar{u}_{n} \bar{c}_{n}(t)\right]
$$

where $\eta(t)$ is the sea surface level, at time $t$, relative to the still water level. The deterministic coefficients $c_{n}(t)$ and $\bar{c}_{n}(t)$, for an advancing vessel, are given by

$$
\begin{gathered}
c_{n}(t)=\sigma_{n} \cos \left(\omega_{e, n} t\right) \\
\bar{c}_{n}(t)=-\sigma_{n} \sin \left(\omega_{e, n} t\right) \\
\sigma_{n}^{2}=E\left(\omega_{n}\right) \Delta \omega_{n}
\end{gathered}
$$

with the wave energy spectrum $E\left(\omega_{n}\right)$ discretised at a number of wave frequencies $N_{0}$. The discretised frequencies are spaced non-equidistantly on the interval $(0 ; 2 \pi]$, i.e. $\Delta \omega_{n}=\frac{2 \pi}{N_{0}-1}$. The present formulation considers a time history of the wave elevation observed from an advancing vessel, which means that the encounter frequency $\omega_{e}$, appearing in the deterministic coefficients, is given by, cf. Eq. (2)

$$
\omega_{e}=\left|\omega_{0}-\omega_{0}^{2} \psi\right|, \psi=\frac{U}{g} \cos \beta
$$

for any given absolute frequency $\omega_{0}$.

\section{Results and discussions}

It has already been introduced that the sole purpose of Algorithm 1 is to transform the encountered wave spectrum, observed on an advancing ship with conditions as listed by Tables 1 and 2 . The test cases are basically represented by three main generating 
spectra being of types: a unimodal Bretschneider spectrum (Cases A-C), a unimodal JONSWAP spectrum (Cases G-I), and a bimodal spectrum (Cases D-F) taken as the sum of two Bretschneider spectra. On the other hand, Algorithm 1 introduces just one single 'scaling spectrum' from which the scaling ratios are calculated (Lines 7-8). Moreover, it is necessary to (pre)assume the 'scaling spectrum' to be of a specific type, independently of the actual generating spectrum (being it of measurements or simulations), since in real-case scenarios the generating spectrum is, of course, unknown. Altogether, it means that specific settings initially must and can be imposed to Algorithm 1, and before the performance of the algorithm is evaluated these settings are pointed out.

\subsection{Specific settings of Algorithm 1}

Scaling spectrum. The scaling wave spectrum (Line 7) is taken as a Bretschneider spectrum that needs as input significant wave height $H_{s}$ and zero-upcrossing period $T_{z}$ (or an equivalent characteristic wave period).

Zero-padding of high-frequency wave components. Generally, wave components with (very) short wave lengths, i.e. large frequencies, possess little energy in relative terms. Therefore, a sort of 'cut-off frequency' is imposed, meaning that the spectral ordinates of the transformed (absolute) wave spectrum (Line 29) are zero-padded when the frequency is larger than $\omega_{0}^{\text {high }}=\pi$. This is equivalent to neglecting waves with a period smaller than 2 seconds. From a numerical point-of-view, it is necessary to introduce the cut-off frequency because, if not, wave energy is, erroneous, transformed at some higher absolute frequencies in region 2, cf. Figure 2. In the general context of wave-ship interactions it is considered acceptable to neglect waves with lengths less than about $6 \mathrm{~m}$, which is the equivalent wave length $\left(L=\frac{2 \pi g}{\omega^{2}}\right)$ on deep water.

Energy conservation. Generally, a small "deficit" of energy is observed for the transformed spectrum. The deficit is in the order $5-10 \%$, and is not merely a result due to the zero-padding of spectral ordinates at higher frequencies but is observed also because of the scaling-procedure itself. Energy preservation can, however, easily be assured by multiplying the transformed spectrum with a ratio based on energy levels. This is possible since, indeed, the true total energy is known from the encounter-wave 
spectrum. Thus, the final version of the transformed spectrum is simply taken as

$$
S_{\text {final }}\left(\omega_{0}\right)=\hat{S}_{\text {final }}\left(\omega_{0}\right)\left(\frac{H_{s}}{\hat{H}_{s}}\right)^{2}
$$

where $H_{s}$ is the significant wave height, obtained from the encounter spectrum, while $\hat{H}_{s}$ is that calculated from $\hat{S}_{\text {final }}\left(\omega_{0}\right)$.

Absolute zero-upcrossing period. The input to the scaling spectrum (Line 7) is significant wave height and zero-upcrossing period, where $H_{s}$ is invariant to the domain, encounter vs. absolute, while $T_{z}$ must relate to the absolute domain. In itself, the latter forms a 'transformation problem' as the zero-upcrossing period, determined from the encounter-wave spectrum, cannot be uniquely converted to the absolute domain for conditions in following waves. A small sensitivity study has, however, suggested a formula to read as given by Algorithm 3 that has the zero-upcrossing period $T_{z, e}$ from the encounter domain as input.

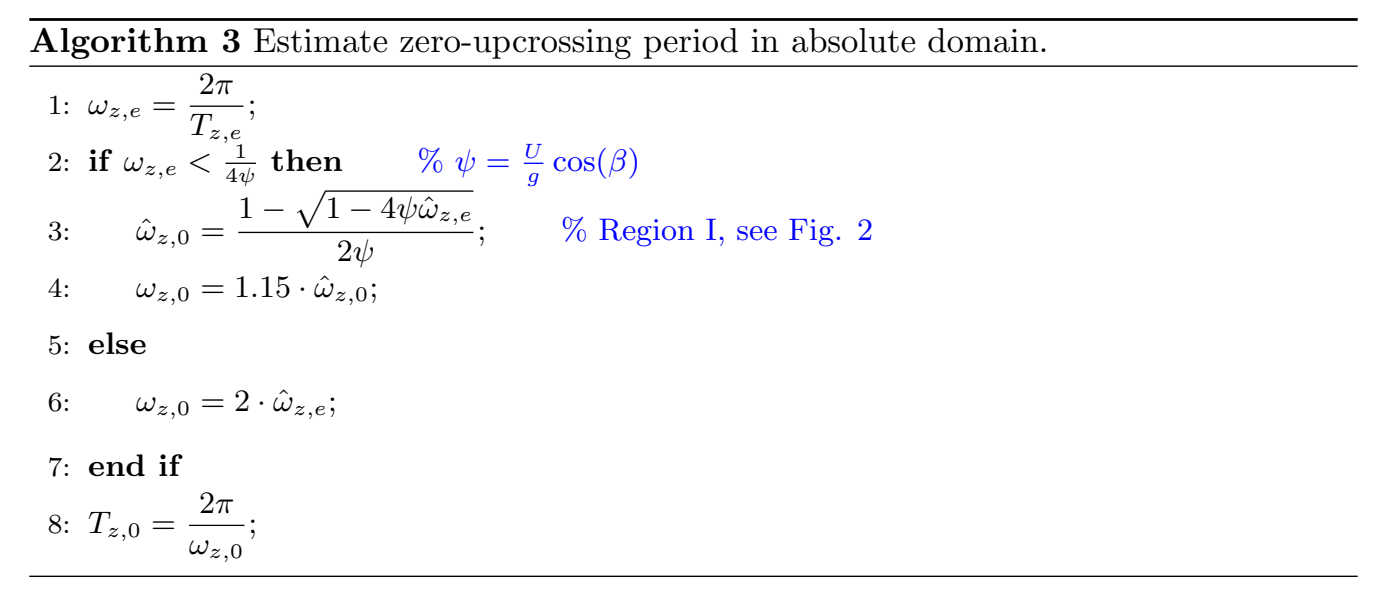

It is important to stress that the zero-upcrossing period obtained from Algorithm 3 is merely a kind of initial estimate used for calculating the scaling ratios (Lines 7-8 in Alg. 1); the 'initial period' is not the final zero-upcrossing period of the transformed spectrum.

\subsection{Spectrum transformations}

The performance of Algorithm 1 can now be tested. In principle, a complete evaluation can be made if the wave spectrum of every single wave elevation is considered. 
Hence, comparisons can be made between sets of wave energy spectra obtained with and without forward speed, respectively, for all wave elevation realisations $(2 \times 20)$ in every listed case (A-I). However, due to space restrictions, only a few detailed evaluations of the transformation algorithm will be presented and, instead, the statistics of the entire set of transformations will be shown. In the following, the results are divided into wave scenarios/cases with a unimodal spectrum (Cases A-C + G-I) and scenarios with a bimodal spectrum (Cases D-F).

Unimodal wave spectrum: A few examples of some detailed comparisons of the complete energy distributions are seen in Figures 8 and 9, where the wave spectrum, observed at the advancing ship in encounter domain, is shown together with the corresponding transformed spectrum in absolute domain. The plots include also the measured, and in the context true, spectrum, produced from the wave elevation records observed at zero-forward speed (in the absolute domain). The considered plots are taken arbitrarily from the ensemble, i.e. twenty realisations in each case, and, although not shown herein, all other plots/realisations show the same general agreement.

The statistics of the spectrum transformations obtained with Algorithm 1 will now be presented. The results are given graphically by bar charts which evaluate the performance of the algorithm in statistical terms. Thus, the charts present the mean value and the standard deviation of characteristic wave periods representing the energy distribution of the (transformed) wave spectrum. Generally, the individual bars/outcomes follow from the statistics of the transformed versions of the original (and true) spectra listed in Table 1 and, for each of the listed cases $(\mathrm{A} 1, \mathrm{~A} 2, \ldots, \mathrm{C} 9+\mathrm{G} 1, \mathrm{G} 2, \ldots, \mathrm{I} 9)$, two bars apply. The one bar is the result based on the spectrum 'measurements', produced from the wave elevation records observed at zero-forward, whereas the other bar is the result based on the transformed spectra, produced from the wave elevation records observed on the advancing vessel; but otherwise the bars are for identical conditions, including the set of uncorrelated, standard normal distributed variables (Eq. 25). Note, onwards the results indicated by the 'measurement bars' are referred to as the true results, while the results indicated by the 'transformed bars' are referred to as the transformed results. The actual charts are concerned with statistics of the wave system's peak period and its zeroupcrossing period, respectively, as these measures, in combination, are good indicators 
of the distribution of wave energy. On the other hand, the total amount of energy, represented by significant wave height, of the wave system is irrelevant to consider since energy conservation is strictly assured/imposed with Eq. (28).

Figures 10-12 show the statistics of all the unimodal cases (A-C + G-I). In addition to the two characteristic periods $\left(T_{z}\right.$ and $\left.T_{p}\right)$, the result of the bandwidth parameter $\varepsilon$ is also included (Fig. 12). In this study the bandwidth parameter is defined by

$$
\varepsilon=\sqrt{1-\frac{m_{0} m_{2}}{m_{4}}}
$$

where the spectral moments are calculated in accordance with Eq. (11), taking note that the frequency and the corresponding spectrum value are those in absolute domain $\left(\omega_{0}\right)$ or encounter domain $\left(\omega_{e}\right)$.

Generally, it is seen from Figures 10-12 that Algorithm 1 performs very well, as the agreements between the true and the transformed integrated parameters, i.e. the agreements between the pairwise sets of bars, are good. This finding applies to the mean values themselves, i.e. the height of the bars, obtained from the 20 wave elevation re-
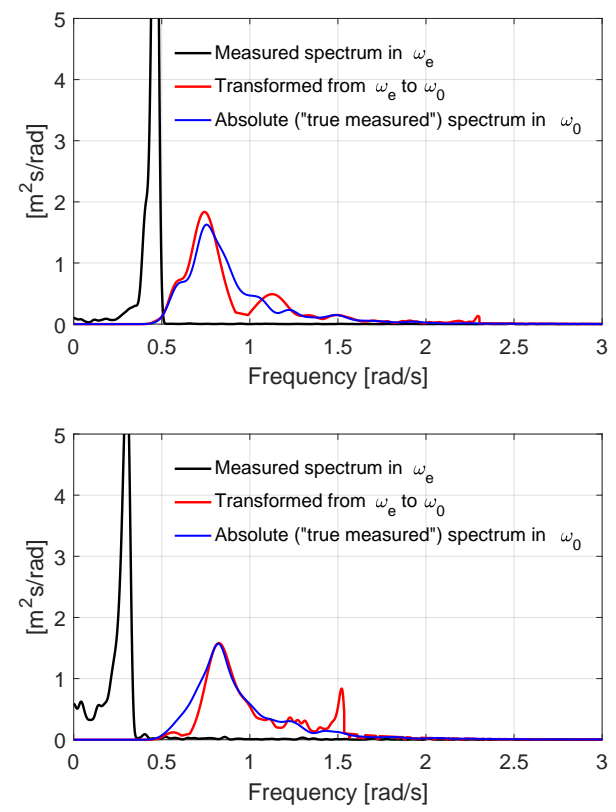

Figure 8: Examples of transformed spectrum considering a unimodal spectrum; from cases A1 (top) and B1 (bottom). Note that the abscissa applies both to encounter frequency and absolute frequency, depending on the considered spectrum. 

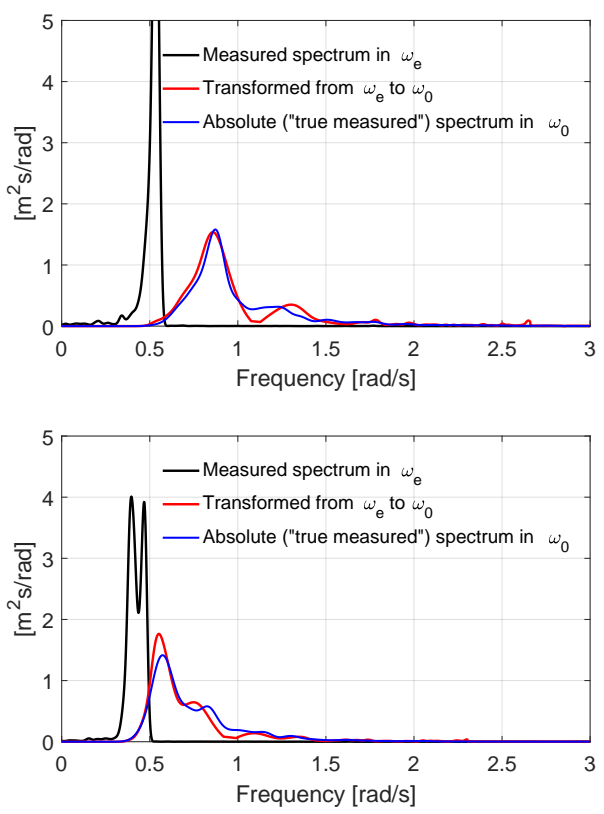

Figure 9: Examples of transformed spectrum considering a unimodal spectrum; from cases G2 (top) and I5 (bottom). Note that the abscissa applies both to encounter frequency and absolute frequency depending on the considered spectrum.

alisations of each case (A-C + G-I), and it applies also to the corresponding standard deviations shown as the red 'error markers' at the top of each bar. Specifically, it is observed that the transformed results for Cases G-I, considering all of the three parameters $\left(T_{z}, T_{p}, \varepsilon\right)$, are not affected by the "inconsistency" in the generating spectrum (being JONSWAP) and the scaling-output spectrum (being Bretschneider). Thus, the agreements between the pairwise results/bars are equally good for the left-hand side plots (Cases A-C) and the right-hand side plots (Cases G-I); keeping in mind that Cases A-C consider wave elevation records generated by use of a Bretschneider spectrum and with the scaling-spectrum being also Bretschneider, while Cases G-I consider wave elevation records generated by use of a JONSWAP spectrum but still with the scaling-spectrum being a Bretschneider spectrum.

Bimodal wave spectrum: The wave scenarios consisting of both wind sea and swells are represented by Cases D-F, see Table 2 (p. 21). Examples of the wave energy distribution in terms of the encountered and the transformed wave spectra of Cases D-F 
are given in Figure 13. Again, the individual plots are taken out arbitrarily from the sets of realisations in each case (here, D1, E1, and F1). Like for the unimodal cases, the energy distributions of the transformed spectra, observed at advance speed, are seen to be in reasonable agreement with the true (measured) spectra, observed at zero-forward speed. Specifically, it is noted that the transformed versions of the encountered wave spectra have the 'main spectral peaks' consistent with the true spectra, although the overall agreement in/between energy distributions are more blurred than found previously for the unimodal
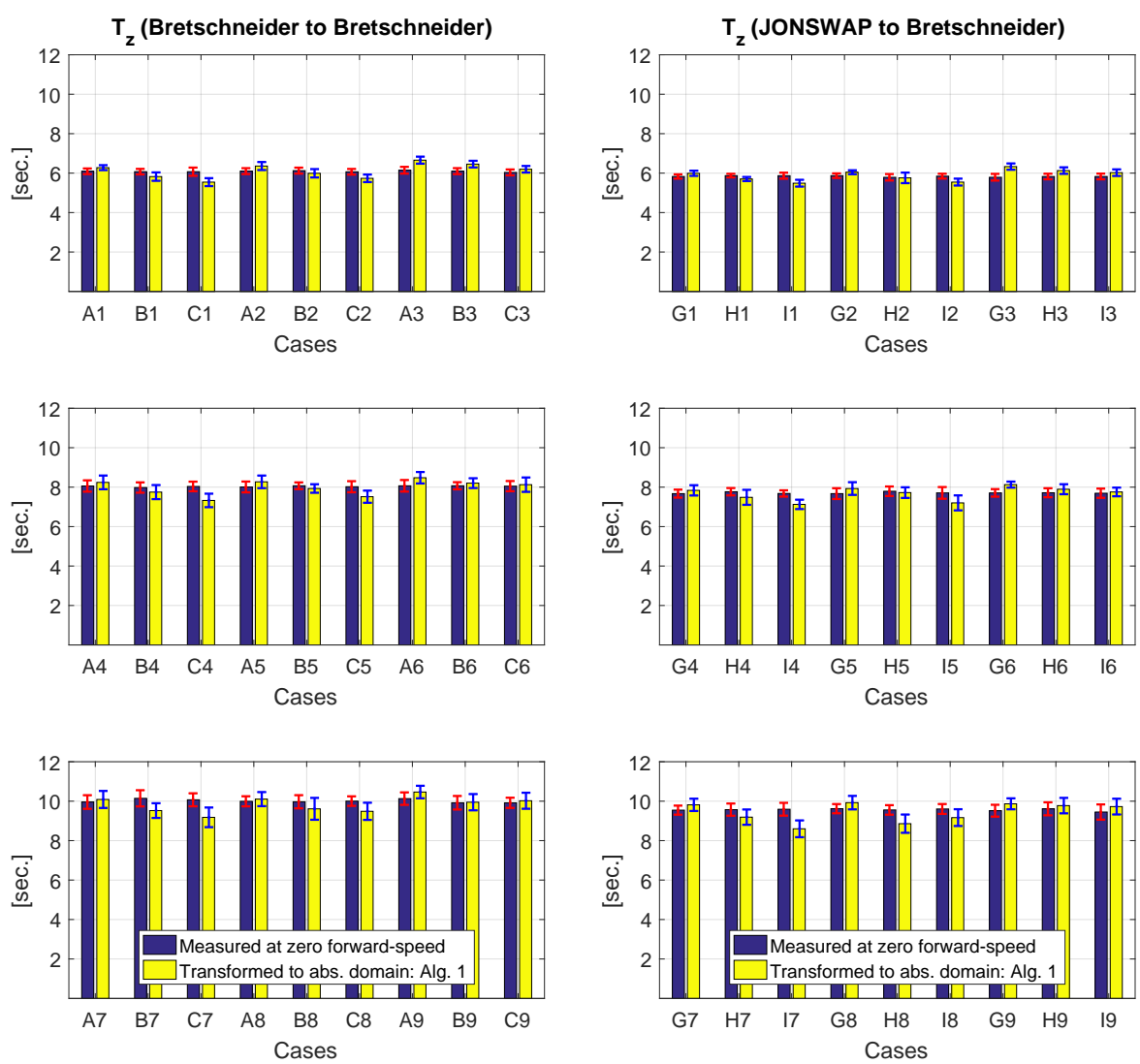

Figure 10: Transformation of spectrum considering a unimodal spectrum. Performance statistics with respect to the zero-upcrossing period; any bar represents the mean value out of the 20 realisations, and the associated pair of error markers represents plus/minus the standard deviation. Left-hand side plots (Cases A-C): Generating spectrum is of Bretschneider type; "output spectrum" is of Bretschneider type. Right-hand side plots (Cases G-I): Generating spectrum is of JONSWAP type; "output spectrum" is of Bretschneider type. 
cases. In this context, it is important to keep in mind that the transformation algorithm (Alg. 1) considers one single scaling spectrum, but still the (correct) transformation of several peaks is possible.

If all realisations for each case (D-F) are considered, the average performance of the transformation algorithm can be statistically evaluated, similar to what was made for Cases A-C + G-I. The results of this evaluation are presented in Figure 14. It is seen that the performance generally are comparable to what was found for the unimodal cases
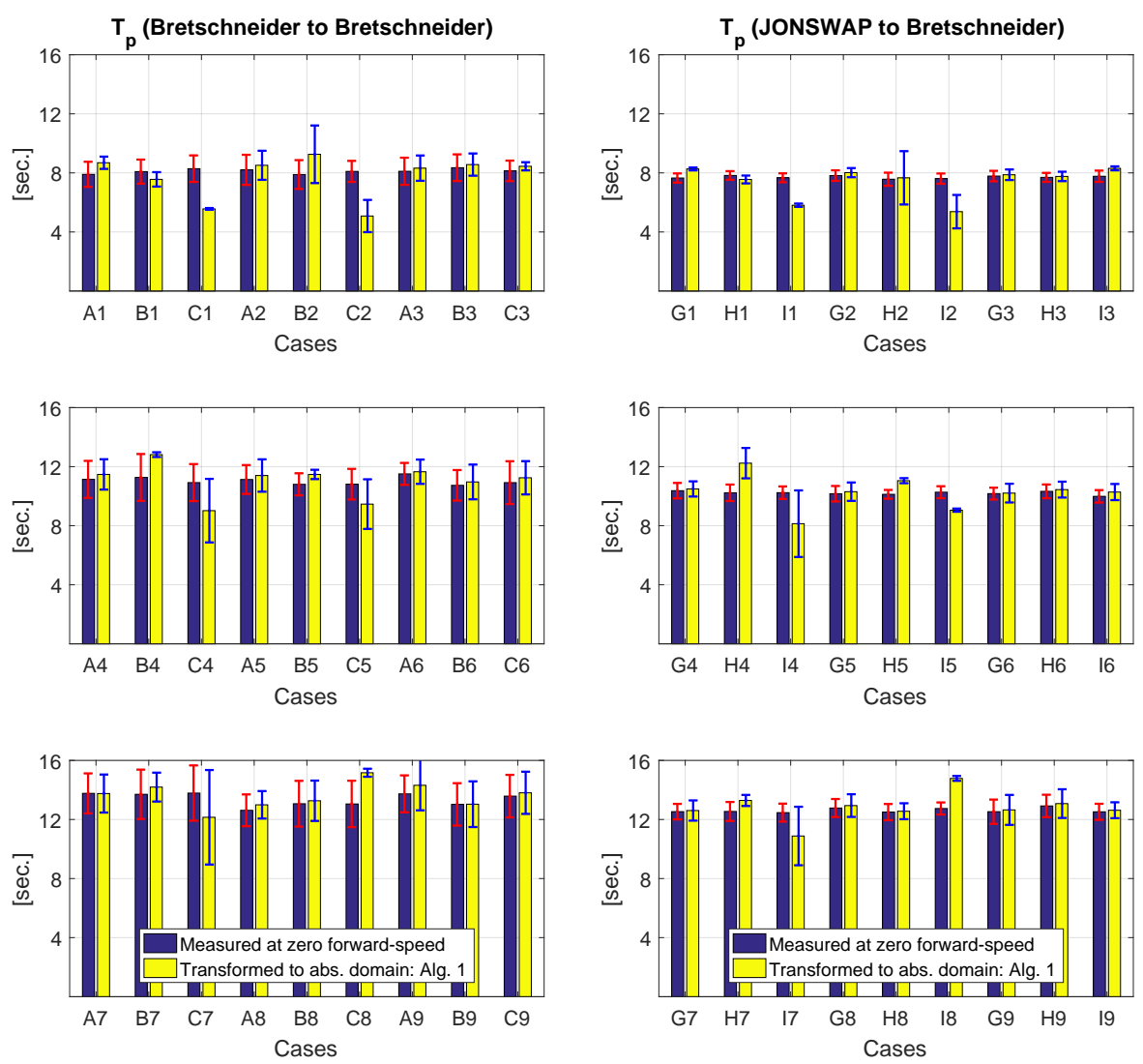

Figure 11: Transformation of spectrum considering a unimodal spectrum. Performance statistics with respect to the peak period; any bar represents the mean value out of the 20 realisations, and the associated pair of error markers represents plus/minus the standard deviation. Left-hand side plots (Cases A-C): Generating spectrum is of Bretschneider type; "output spectrum" is of Bretschneider type. Right-hand side plots (Cases G-I): Generating spectrum is of JONSWAP type; "output spectrum" is of Bretschneider type. 
(Figs. 10-12); a finding that applies to all parameters $\left(T_{z}, T_{p}, \varepsilon\right)$ both with respect to the mean values (the height of the bars) and the spreading around it (the error markers). Thus, the transformation algorithm (Alg. 1) can be successfully applied to transform also a bimodal spectrum from encounter to absolute domain.
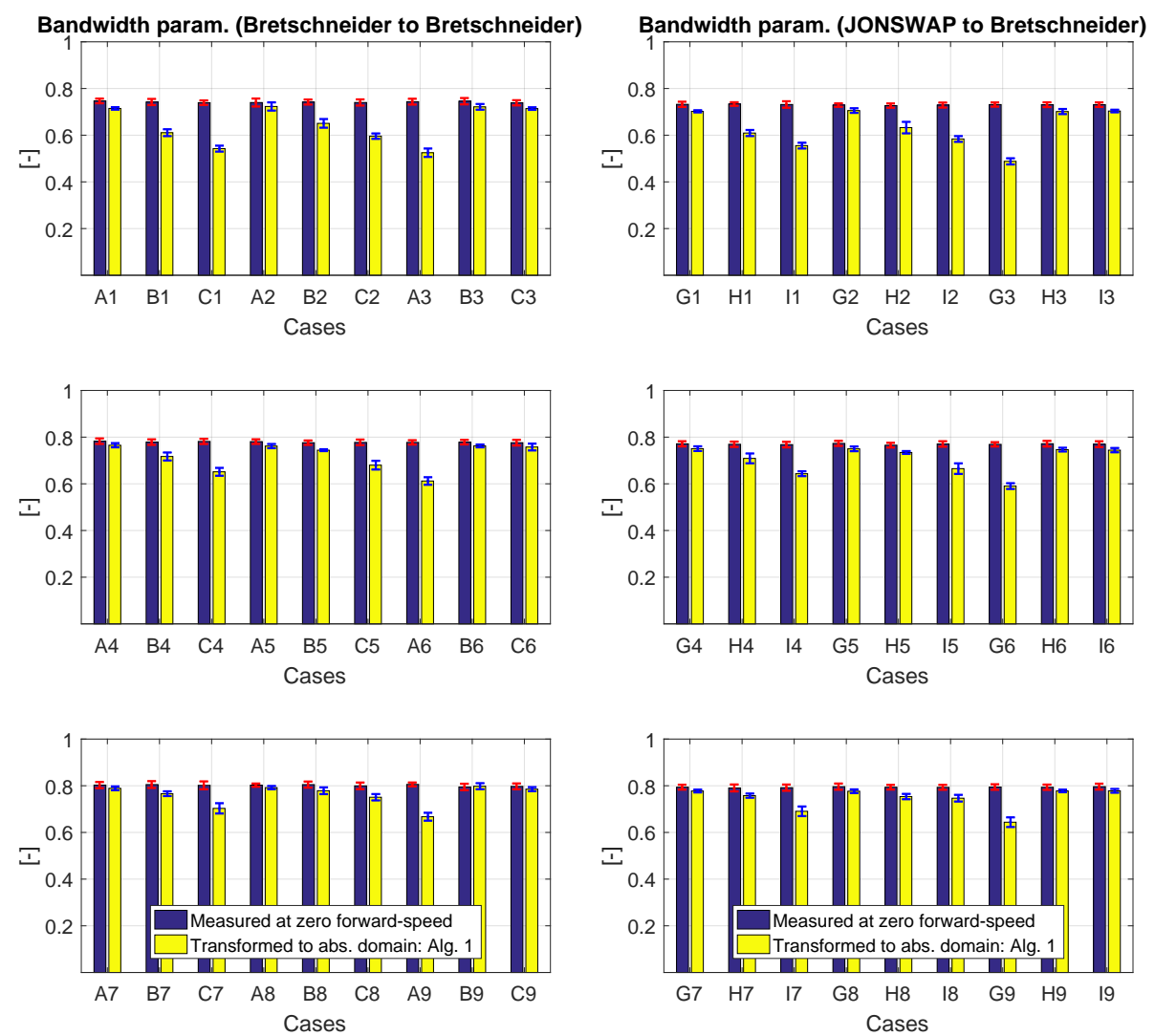

Figure 12: Transformation of spectrum considering a unimodal spectrum. Performance statistics with respect to the bandwidth parameter; any bar represents the mean value out of the 20 realisations, and the associated pair of error markers represents plus/minus the standard deviation. Left-hand side plots (Cases A-C): Generating spectrum is of Bretschneider type; "output spectrum" is of Bretschneider type. Right-hand side plots (Cases G-I): Generating spectrum is of JONSWAP type; "output spectrum" is of Bretschneider type. 


\section{Conclusions}

For practical reasons, it is often necessary to transform an observed/estimated wave spectrum from an encountered domain to the absolute domain, where the former, in this context, is the domain in which observations are made on an advancing ship. The absolute domain, on the other hand, is that in which the observer is at a fixed position relative to the inertial frame wherein the (progressing) waves are described. The transformation of a wave spectrum in the particular direction, from encounter to absolute domain, is
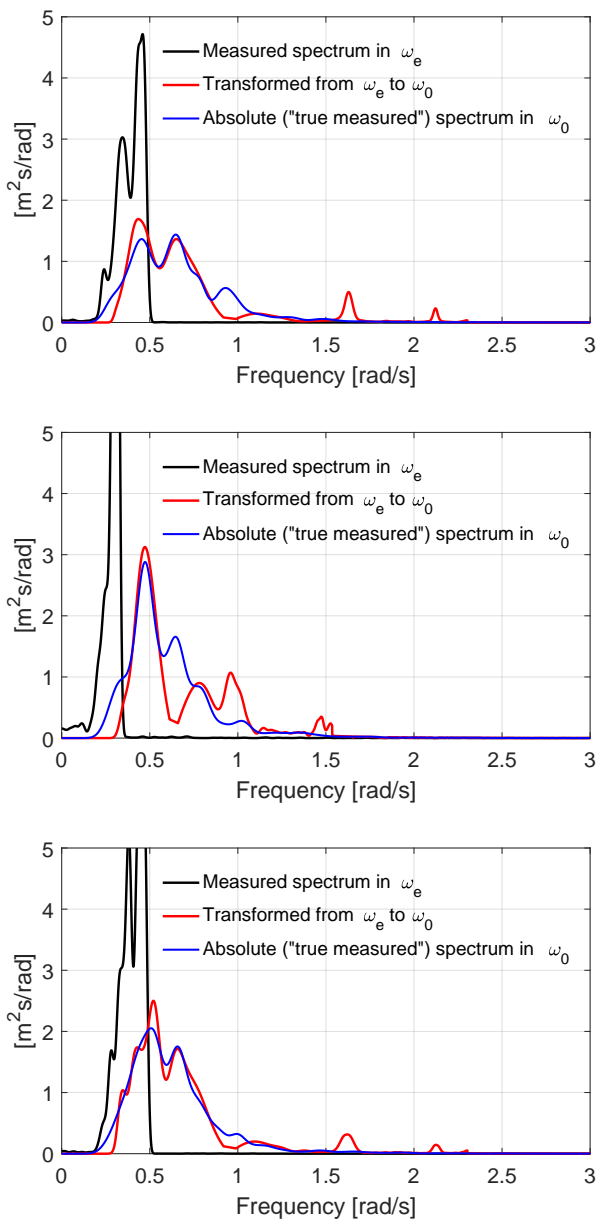

Figure 13: Examples of transformed spectrum considering a bimodal spectrum; from cases D1 (top), E1 (middle) and F1 (bottom). Note that the abscissa applies both to encounter frequency and absolute frequency, depending on the considered spectrum. 
trivial if the ship meets the waves at an angle from beam to head sea, but there exists no unique solution when the vessel advances in following waves $(\beta=[0-90[$ deg.).

The article has proposed two specific (pseudo) algorithms that offer the possibility to make a mathematically consistent transformation. The one algorithm, Algorithm 1, is based on a scaling approach, where a certain parameterised wave spectrum must be assumed for calculating scaling ratios of wave spectral densities. The scaling ratios apply to specific absolute frequencies, obtained through the Doppler Shift of given encounter
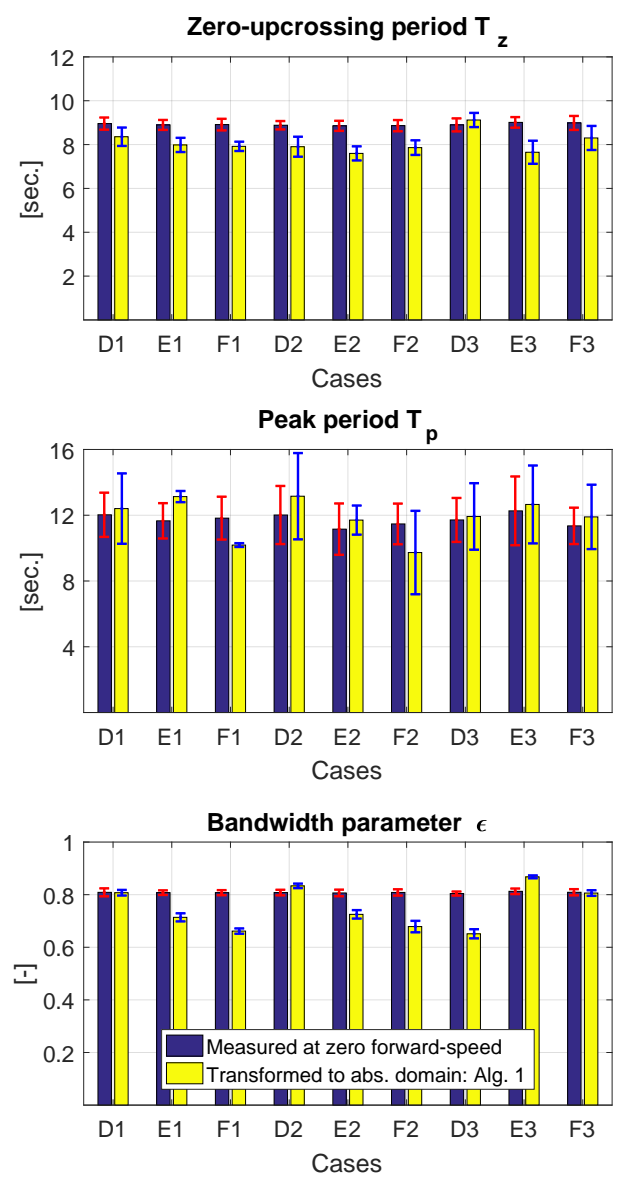

Figure 14: Transformation of spectrum considering a bimodal spectrum. Performance statistics with respect to characteristic periods $\left(T_{z}\right.$ and $\left.T_{p}\right)$ and bandwidth parameter; any bar represents the mean value out of the 20 realisations, and the associated pair of error markers represents plus/minus the standard deviation. Generating spectrum is of a double-peaked Bretschneider type; "output spectrum" is of Bretschneider type. 
frequencies, and, hence, a transformed spectrum becomes readily available. The other algorithm, Algorithm 2, relies on an optimisation problem aiming at the fitting of a parameterised wave spectrum.

The study showed that Algorithm 1 could successfully be used to transform an encountered wave spectrum to the absolute domain. In the study, tests were made with many different wave systems of both unimodal and bimodal type, and transformations could be made reasonably accurate for different advance speeds. Algorithm 2 could also be used to make the transformations, but it was a problem of the algorithm that the solution turned out to be quite sensitive to the initial guess of the wave parameters being optimised. It is therefore left as a future exercise to improve Algorithm 2.

\section{Acknowledgement}

This work has been supported by the Research Council of Norway through the Centres of Excellence funding scheme, Project number 223254-AMOS.

\section{References}

